\title{
Towards a sustainable implementation of interoperability solutions: bridging the gap between interoperability requirements and solutions
}

\author{
Nicolas Daclin ${ }^{1}$, Sihem Mallek Daclin ${ }^{1}$ \\ ${ }^{1}$ LGI2P, Parc scientifique G. Besse - école des mines d'Alès, 30035 Nîmes cedex, France \\ name.surname@mines-ales.fr
}

\begin{abstract}
The main objective of this communication is to present and discuss the need, for partners, to suitable interoperability solution according to their expectations. First, the problematic of the selection of a solution is presented and the stakeholders' needs to tackle this statement are highlighted. Then, existing works related to interoperability requirements and interoperability solutions are briefly presented and discussed. Finally, criteria - and associated examples - that guide stakeholders in their selection are presented.
\end{abstract}

Keywords: Enterprise interoperability engineering, verification, interoperability requirements, interoperability solutions.

\section{Introduction}

The concept of interoperability [1] is now considered as a crucial issue and a key factor of success for such enterprises that share and exchange processes, services data, enterprise applications [2]... in a collaborative context. Numerous works has been led to characterize [3], implement [4] [5] and improve [6] [7] this aspect of a partnership. Originally coming from the computer science and Information and Communication Technologies (ICT) fields [8] and, exclusively considered as a technical problem, interoperability has expanded, since, and considers now other less technical aspects. Also, in addition to technical aspects, interoperability can includes organisational aspects (for instance, who does what, how, when and who is responsible for what?...) as well as conceptual aspects (for instance, does the data to be exchanged use a data model?...) [9] [10] [11]. Similarly, if interoperability was seen only as an ability that enables and improves the sharing and the exchange of data, it now considers other fields such as the interoperability of processes (synchronization / coordination of activities, mutual adjustment [12]). Finally, as a simple matter of interfacing issue, the development of interoperability takes also an interest in other aspects of a collaboration that can affect either a given partner or the partnership itself. In that sense, it takes, for instance, an interest in the preservation of the autonomy of each partner involved into the collaboration [13].

As we may have noticed, interoperability has become an important expected capability - in other word a requirement (especially a non-functional requirement - 
NFR [14]) - for enterprises that want to establish and maintain an effective partnership in terms of exchange and sharing of their information, products and resources (material as human)... but also that want to align and, further, if requested, to orchestrate their process commonly or else, that want to work together at an higher level in term of decision or policies. However, with a better understanding and definition of interoperability, also appeared a greater complexity in its implementation, monitoring and control and improvement. Naturally, interoperability solutions - that cover the set of interoperability aspects - are developed to satisfy the needs of interoperability for partners that collaborate. In this context it is important to allow partners to select solutions that meet - at best - all their expectations in term of interoperability. Although the space of problems of interoperability is clearly identified on one hand, and the space of interoperability solutions is clearly defined on the other hand, the link between both is not clearly established and formalized. This statement may lead partners to select their interoperability solutions in a hazardous way and without knowing the potential impacts of the implementation of a given interoperability solution onto other aspects (e.g. other requirements, performance of the collaboration...) of the partnership. As a consequence the here presented research attempts to clarify the link between interoperability requirements and interoperability solutions and to enable partners to select solutions fully adapted to their needs and in a proper way.

This paper is structured as follow. After this brief introduction, the problem and expected results of this research are presented. The research works related to the fields of this research is given and discussed in section 3. Section 4 presents the foundations of the proposed reference model to define and select accurate interoperability solutions regarding the dimensions that has to consider. To illustrate the first proposition to implement and to use the proposed model, a case study is made available to the readers and shows it into the overall context of interoperability within a collaborative process. The final section presents the conclusions and the prospects of this research.

\section{Problem and expected outcomes}

In enterprise collaboration engineering context as in any other domains (system engineering, Information System, mechatronic...), it is difficult for stakeholders to have and to select a solution that satisfies all their expectations initially expressed. Indeed, it is not enough to have a jumbled and unconnected list of solutions if it is not consistently and precisely related with a set of requirements and if the potential impact that can be caused by solution onto its environment is unknown (Figure 1) and vice and versa. Thus, "there is no interest to identify the problems if there are no adapted solutions just as there is no interest to give solutions if the problems are not clearly identified". 


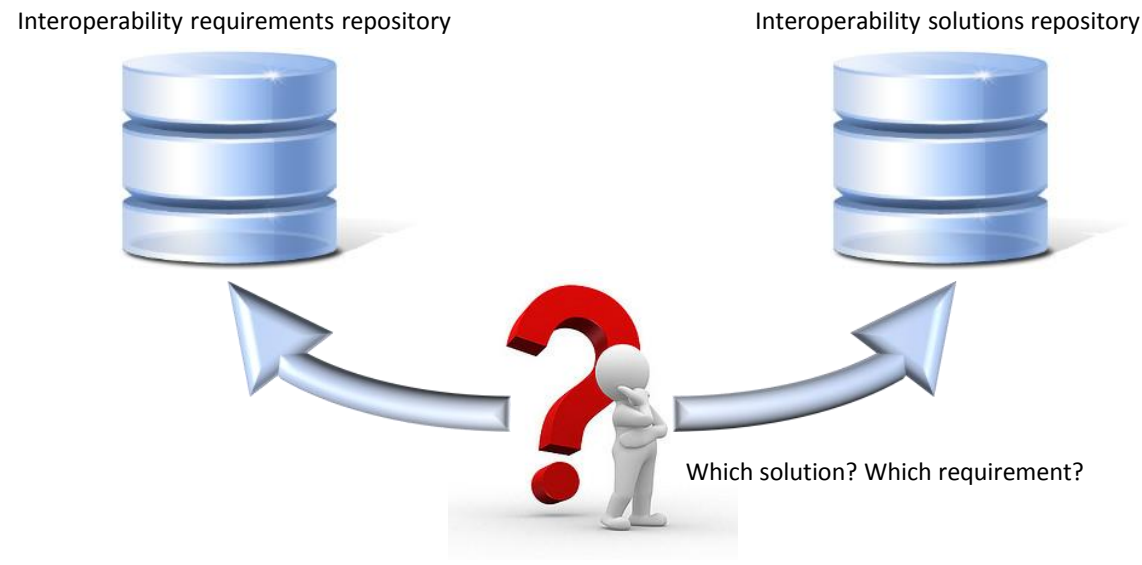

Fig. 1. The selection of solution dilemma

First, for a given expectation, several solutions can be available. However, all relevant solutions are not necessarily known and, naturally, the stakeholders favor the solution they control without further investigation and at the risk that such solution covers partially the problem or, in the worst case, is not adapted to the problem in the end (in operational phase for instance). To face up this problem, all solutions have to be (1) fully and clearly identified in order to avoid to stakeholders to miss out on a relevant solution and (2), mirrored from the (or the set of) need(s) they allow to satisfy. That means to make available a relevant structure in which requirements and solutions are properly related and in agreements with the considered problem namely the ability to interoperate whether conceptually, technically or else, organizationally and during all the life cycle of the partnership.

Second, a given solution, and this independently of the covered field, is developed to satisfy a need. However, it is often forgotten that a solution can fully match an (or a set of) expectation but at the same time, this one can have an impact on other expectation and further on the system to be developed. In that case, the problem is not reduced to the choice of "the" solution but to choice of "the best" solution that means the solution which will satisfy the need for which it is developed but also the solution for which its impacts on the context will be fully identified and defined to allow stakeholders to choose it in confidence. Thus, beyond to have a set of solutions related to a set of requirements as mentioned before, it is also required to guide the stakeholders in their process of selection according to identified criteria that characterize the solution precisely (for instance in term of impact on other requirements).

\section{Interoperability requirements and solutions: a quick scan}

Numerous works deal with interoperability requirements for collaborative enterprises on the one hand and with the development of interoperability solutions - 
either conceptual technical or organizational - on the other hand. However, few works are concerned with the definition of the relation that can exist between the world of interoperability requirements and the world of interoperability solutions.

First, the world of interoperability requirements engineering focuses on two aspects. On the one hand, it is about to identify, to define precisely [15] [16] and writing requirements [17] in order to allow their verification - by stakeholders - either by the way of automatized and formal techniques (e.g. model checker) or not (e.g. test, expertise...). On the other hand, it is about to classify the interoperability requirements to ensure their management and traceability [18]. This aspect is strongly based on existing categorizations that are proposed by interoperability frameworks (Figure 2).

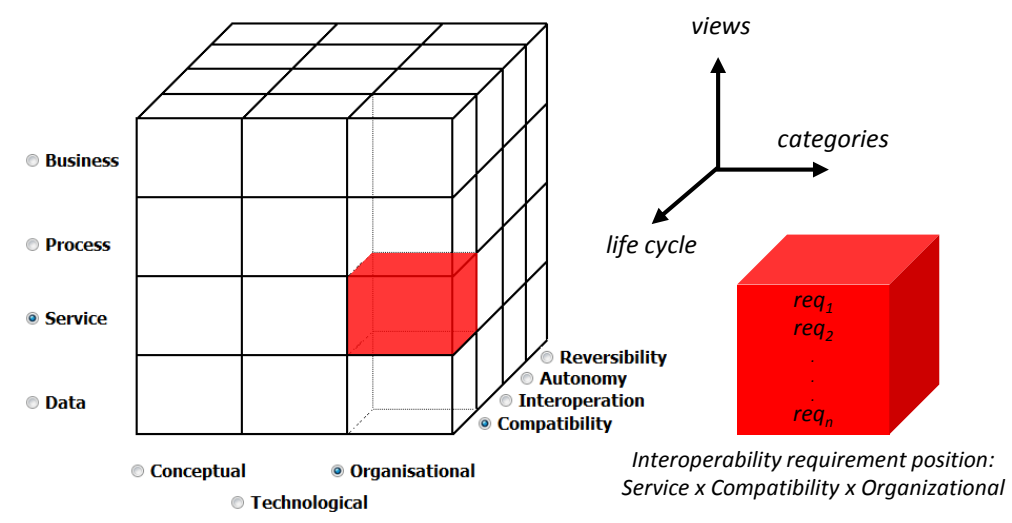

Fig. 2. The Framework for enterprise interoperability requirements

These two aspects are fundamentals since the principle of requirements engineering is that, the more the requirements are accurately identified the more a solution fully adapted can be found. However, if the consideration of the interoperability as requirement is accepted and widely studied, the connection with existing interoperability solutions is weak and their choice is often at the discretion of the stakeholders without any guidelines or support.

Second, some approaches make interoperability solutions available and rely strongly on the structuration of interoperability through interoperability frameworks. Let's mention the enterprise interoperability framework [13] that proposes no less than 66 interoperability solutions sorted onto its three axes such as interoperability concerns, interoperability approaches and interoperability categories [19]. However, the exploitation of this framework is difficult and does not allow stakeholders to know if a given solution is fully adapted to their interoperability needs since (1), they don't know the possible impact (and its evaluation) on the environment, (2), several solutions can be proposed for a given intersection between axes, (3), they don't know if a selected solution does not go beyond their effective needs (some solutions cover several categories and concerns that are not necessarily interesting for partners) and (4), these solutions are not formally related to requirements since those latter are not 
clearly and explicitly formulated i.e. following a structured and accepted format [23]. Thus, although a large number of solutions is proposed, this remains too general in terms of guide in the choice of solutions (not related to specific needs) and without precision this large sample can lead stakeholders to select " $a$ " solution and not "their" solution (Figure 3). In the same vein, let's mention also [20] that proposes a state of the art on enterprise interoperability services (business, semantic, data...).

\begin{tabular}{|l|l|}
\hline Name of solution, author & \\
\hline Interoperability concern & To choose in the interoperability framework \\
\hline Interoperability barrier & To choose in the interoperability framework \\
\hline Interoperability approach & To choose in the interoperability framework \\
\hline Interoperability problem & Short and precise description of the knowledge \\
\hline Interoperability knowledge & $\begin{array}{l}\text { Description of the knowledge vs. problem } \\
\text { Contribution of the knowledge to remove the barrier }\end{array}$ \\
\hline Example (optional) & $\begin{array}{l}\text { To illustrate the use of the knowledge through a concrete } \\
\text { example }\end{array}$ \\
\hline Remark & Open issues and limitations \\
\hline References & List of references \\
\hline
\end{tabular}

Fig. 3. Template for interoperability solution proposal [19]

Other approaches propose a set of solutions - according interoperability aspects but without necessarily be positioned precisely into a framework. In this case, let's mention [21] that proposes interoperability solutions (strategic interoperability, process interoperability, technical interoperability and semantic interoperability) for SMEs. This kind of approach grasps the necessity to consider a solution not only as a simple answer to a problem but to also to position it into a context allowing to consider other aspects that can be linked to this one. Thus, it examines and proposes solution according to interoperability such as technical, process-based and semantic but also taking into account characteristics - allowing to evaluate their relevance and usability for an SME (from high relevance to not applicable) - such as:

- Startup-costs: it represents the cost to implement technology or standard. This cost has to be as inexpensive as possible for SMEs.

- Running costs: SMEs are not able to afford high running costs for their interoperability solutions. This cost has to be, also, as inexpensive as possible for SMEs.

- Implementation effort: usually SMEs do not possess IT-system technically advanced as well as they do not employ IT-experts. Thus interoperability solution has to be easy to implement that means without strong efforts and advanced skills.

- Direct use for an SME interoperability solution: this aspect is concerned by the evaluation of the use of an interoperability solution that fulfills the needs of SMEs. 
- Conceptual input for an SME interoperability solution: interoperability solutions are also analyzed in term of conceptual input for further researches on interoperability for SMEs.

Thus, this approach does not stop to the simple proposition of a set of interoperability solutions but, because of the specifics of SMEs, it has evaluated a set of interoperability solutions (BFC, BPMN, SAP...) according to defined criteria and it has provided an analysis allowing to guide SMES in their choice of interoperability solutions.

From these considerations, it appears that interoperability requirements on the one hand and solutions for interoperability on the other hand are clearly identified, but the link between them is not obvious or, at least, fully weaved. However the underlying difficulty remains the same for stakeholders: to select the better solution for their needs. In this way, it is required (1) to link solutions and requirements and (2), to highlight criteria that guide stakeholders in their choice of interoperability solution when one of them is identified as relevant to meet expectations.

\section{Reference model for interoperability solutions}

The reference model for interoperability solutions is a structure that embeds parameters aiming to make a set of solutions available (this set is fully related to those proposed in the enterprise interoperability framework [13]) according to a (or a set of) requirement considered and positioned in agreements with the dimensions of the enterprise interoperability requirements framework (interoperability views interoperability lifecycle - interoperability problems) [18]. However, as mentioned hereinbefore, the simple relation "requirements-solutions" is not sufficient to define accurately if a solution is really a well candidate solution regarding to interoperability project between partners and more broadly the partnership itself. Indeed, although a given solution allows to meet a requirement satisfaction, its implementation can have collateral effects on several parameters. As a consequence these parameters have to be integral part in the choice of an interoperability solution. To this purpose, the proposed model includes four parameters such as: interoperability problems resolution, solution overview, other requirements impact, and granularity impact.

Interoperability problems resolution. This parameter allows stakeholders to know precisely which problem(s) the proposed solution can solve in term of interoperability. These highlighted solutions depend not only on the resolved problem but also on fields covered in enterprises and the phase of the partnership. Thus the solution is positioned in agreements with the dimension of the framework for interoperability requirements that means according to (1) the interoperability views that represent the enterprises' domains that can be impacted by a problem of interoperability (business, process, service and data / resources / material), (2) the interoperability lifecycle levels that represent the moment of the partnership at which interoperability is requested by partners (e.g. the beginning of the partnership involves solutions that come under interfacing and thus, of compatibility) and (3), the abstraction levels that represent the categories of interoperability that can be developed in enterprise and thereby the problems of interoperability (conceptual, 
organizational ant technical). It is to note that a given solution can cover several interoperability views or else, interoperability life cycle levels. For instance solution such as semantic annotation can be used for each levels and can be implemented at the beginning of the partnership to solve existing - and avoid future - problems of misunderstanding (of models, data...). Solution such as Business Process Modeling and associated workflow engine are suitable for the process level to solve organizational and technical problems and can be implemented at the beginning of the partnership in order to align processes and useful for interoperation phase by the execution of the common process.

Solution overview. This parameter takes into consideration the general information about the proposed solution. It makes available a quick overview of the solution and allows stakeholders to identify easily relevant solutions from those that are not adapted (e.g. solutions previously implemented without success). To this purpose, it gives information about the name of the solution (not limited to its acronym, source of misunderstanding), applications that are possibly existing and that support the solution (e.g. BPMN modeler software to support processes modeling and execution), average cost (or range) for implementation, average time (or range) to implement the solution and, material and human means requested to implement the solution.

Other requirements impact. A solution is implemented to satisfy one (or a set of, for a solution that covers several aspects of interoperability) interoperability requirement. However, if a given solution allows to improve interoperability it can also induce a degradation by impacting requirements that are related to its application domain. For instance, let's consider the requirement defined such as "if task requires aptitude then resource has aptitude" that has to be satisfy when resources (human, material, software) are shared by partners. This requirement affects the domain of task, resources and aptitude. The non-satisfaction of this requirement can lead, at best, to a bad achievement of the considered task and, at worst, to its non-achievement. A solution could be the replacement of the allocated resource by another one that has the requested aptitude or to train the current resource for the task. However, the choice of a solution (e.g. changing of resource) can impact the satisfaction of other requirement, in this case, those ones that take an interest in the realization of other tasks and the availability of the shared resources (e.g. "It is possible that task is starting and resource is available"). It is to note that the impact is not limited to other interoperability requirements but includes also other ones (functional as well as nonfunctional, e.g. performance requirements). For instance, the possible solution previously proposed can impact the duration of the realization of the task. As a consequence, when an interoperability solution is developed or proposed, it has to highlight which other requirements can be impacted.

Granularity impact. It represents the level of detail of the object impacted by interoperability. The more precise the identification of the object impacted by interoperability is, the more the solutions selected and implemented by partners will be fully adapted and efficient. Indeed, interoperability can impact a partnership (mission, objectives...) as well as a given partner (mission, objectives but also component, resources...). For instance, the requirement "partners have necessary authorization to access shared data" has an impact on the good unwinding of the activities of the partnership (loss of time to access the information required to perform an activity). On the other side, the requirement "function $f$, performed by resource $r$ 
involved in partnership, is even though performed" has a direct impact on a partner (precisely the execution of a function). From these knowledge, partners can select / adapt interoperability solutions or, possibly, relax requirements. As a consequence, the interoperability requirements (as other ones) are a part of a whole and their impacts must not be considered solely as a local matter but also as an overall matter.

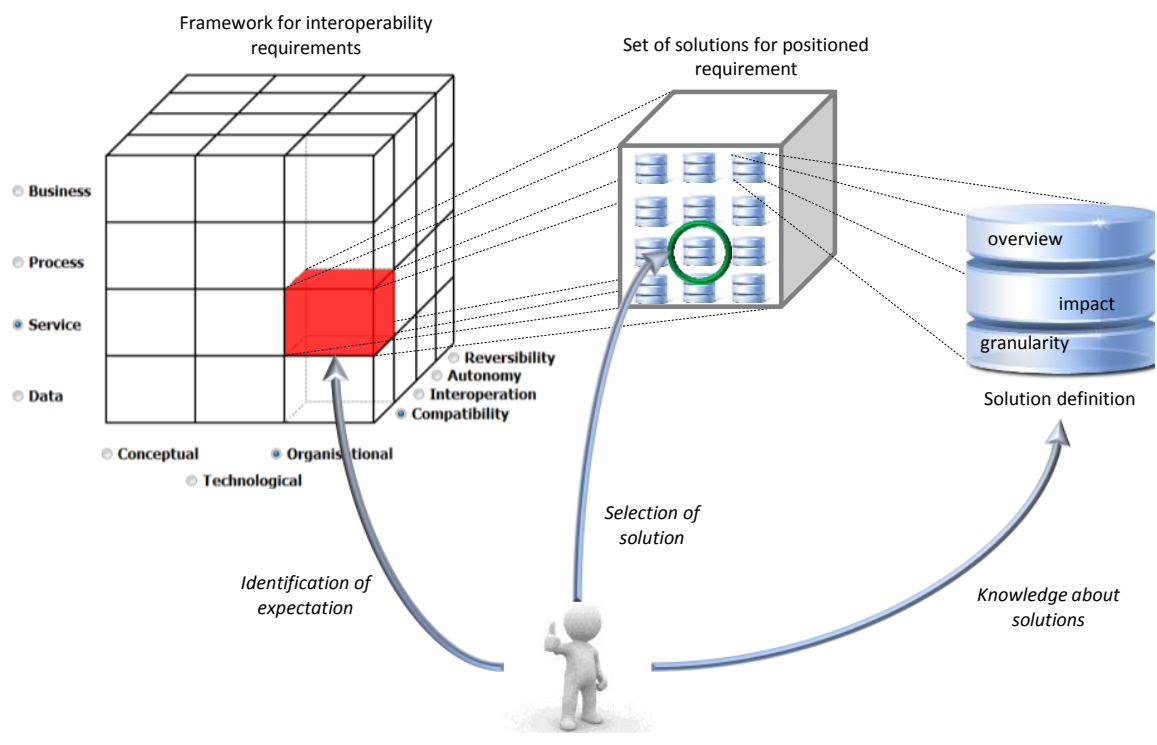

Fig. 4. Reference model for interoperability solution.

Finally, an important aspect of the model is the knowledge of the impact of a solution onto other requirements and stakeholders. Beyond the simple knowledge of the impacted elements, it is important for partners to know in which measure they are impacted. To this purpose, it would be interesting to quantify it or, at least, to define a trend showing the importance of the impact in order to guide more precisely stakeholders in their choice. Moreover, regarding the choice, itself, it would be interesting to rely on existing tools used in Systems Engineering to select a candidate solution [22]. Indeed, a solution is characterized in the proposed model with different parameters (impact granularity, cost/time to implement...) and the use of these kind of tools allow to weight them and implement mechanisms that partially automatize the selection.

As a case study, the drug circuit process, available online at goo.gl/chclk7, shows a possible first implementation of the proposed model of interoperability solutions (partially since the proposed model has to be validated). This illustration focuses on the verification of interoperability requirements - written or selected by stakeholders but the paper does not consider this problematic - and the proposition of adapted solution according to the unsatisfied requirement, the potential interoperability 
problem as well as the proposed solution and its possible impact on other requirements if it is implemented.

\section{Conclusion and prospects}

In a collaborative context, having mechanisms allowing to choose fully adapted interoperability solutions is a serious benefits, for enterprises, in term of costs for implementation and time to launch work. Furthermore, beyond the simple interoperability capabilities, benefits have an impact on the functioning of the whole partnership. The paper has presented a first proposition of a model that links interoperability requirements and interoperability solutions and, further, attempts to define a set of parameters allowing to guide stakeholders finely and accurately in their selection of a solution.

The advantage of such model is to guide precisely stakeholders in their choice rather than to have a sample list leading, too often, to select solutions unsuitable for their requirements. Obviously, parameters can be added depending on the special features of requirements (special needs in term of interoperability) or on the special features of partnership. However, having a link between requirements and solutions as well as general parameters characterizing them can be used as a base of selection.

Finally, it is to note this model - after its validation - will have to be integrated and technically implemented with existing tool and approach allowing to manage the lifecycle of an interoperability requirement from its elicitation and writing to the selection of a an adapted solution to satisfy it and via its verification [15].

\section{References}

1 ISO (2011). Advanced automation technologies and their applications - Part 1: Framework for enterprise interoperability, International Organization for Standardization, ISO 11354, ISO/TC $184 / \mathrm{SC} 5$.

2 ISO, «ISO 15745 - 1, Industrial automation systems and integration - Open systems application integration framework - Part 1: Generic reference description »,ISO TC184/SC5, 2003

3 Chen D., Doumeingts G., Vernadat F. Architectures for enterprise integration and interoperability: Past, present and future. Computers in industry, vol. 59, issue 7, pp. 647659, 2008.

4 Daclin N., Chen D., Vallespir B. Developing enterprise collaboration: a methodology to implement and improve interoperability, Enterprise Information Systems, DOI: 10.1080/17517575.2014.932013, 2014..

5 Bourey J.P., Grangel R., Doumeingts G., Berre A. J. Report on Model Driven Interoperability, INTEROP Network of Excellence IS 508011, deliverable DTG 2.3, 2007.

6 C4ISR Architecture Working Group (AWG). Levels of Information Systems Interoperability (LISI), USA Department of Defense, 1998.

7 Ford C.T. Interoperability measurement, PhD Thesis, Department of the Air Force Air University, Air Force Institute of Technology, 2008.

8 IEEE. 1990. IEEE Standard Computer Dictionary: A Compilation of IEEE Standard Computer Glossaries. New York: Institute of Electrical and Electronics Engineers. 
9 Wang, X., Xu, X. W. DIMP: An Interoperable Solution for Software Integration and Product Data Exchange. Enterprise Information Systems 6 (3): 291-314, (2012).

10 Jardim-Goncalves, R., A. Grilo, C. Agostinho, F. Lampathaki, and Y. Charalabidis. Systematisation of Interoperability Body of Knowledge: The Foundation for Enterprise Interoperability as a Science. Enterprise Information Systems 7 (1): 7-32, (2013).

11 Panetto, H., and J. Cecil. 2013. "Information Systems for Enterprise Integration, Interoperability and Networking: Theory and Applications." Enterprise Information Systems 7 (1): $1-6$.

12 Vallespir, B., Chen, D., Ducq, Y. Enterprise modelling for interoperability. 16th IFAC World Congress, Prague, Czech Republic, july 2005.

13 Chen, D., Dassisti, M., Elveaeter, B.: Enterprise interoperability framework and knowledge corpus - final report. Interop deliverable DI.3, may (2007).

14 Willis J. Systems Engineering and the forgotten '-Illities, 14th Annual Systems Engineering conference, San Diego, CA, the United States of America, 2011.

15 Mallek S., Daclin N., Chapurlat V. The application of interoperability requirement specification and verification to collaborative processes in industry, Computer in Industry, vol.63, issue 7, pp. 643-658, 2012.

16 The Open Group Architecture Framework. TOGAF version 9, chapter 29 Interoperability requirements, 2011.

17 Open Management Group (OMG): Semantics of Business Vocabulary and Business Rules (SBVR) - version 1.0, available online at http://www.omg.org/spec/SBVR/1.0/PDF, 2008.

18 Daclin N., Mallek S. Capturing and structuring interoperability rquirements: a framework for interoperability requirements, $7^{\text {th }}$ International Conference on Interoperability for Enterprises Systems and Applications (I-ESA'14), Albi, France, 24-28 March 2014.

19 Chen, D., Dassisti, M., Elveaeter, B.: Enterprise interoperability framework and knowledge corpus - final report - Annex: Knowledge Pieces. Interop deliverable DI.3 - Annex, may (2007).

20 Elvesæter B., Gorka Benguria G., Capellini A., Del Grosso E., Taglino F. State-of-the-Art and Baseline EI Services Specifications. COIN Project, Deliverable D5.1.1, 2008.

21 Balzert S., Burkhart T. Werth D., Laclavik M., Seleng M., Mehandjiev N., Carpenter M., Stalker I. D. State of the art solutions in enterprise interoperability. Enterprise information systems for business integration in SMEs: Technological, Organizational and Social dimensions, chapter 12, Maria Manuela Cruz-Cunha eds., 2012.

22 Couturier P., Lô M., Imoussaten A., Chapurlat V., Montmain J. Tracking the consequences of design decisions in mechatronic Systems Engineering. Mechatronics, vol. 24, issue 7, pp. 763-774, 2014.

23 INCOSE. Guide for writing requirements, v1.0, Requirements WG-INCOSE, INCOSE-TP2010-006-01, 2012. 